\title{
Combination Analgesic Development for Enhanced Clinical Efficacy (CADENCE Trial): Study Protocol for a Double-Blind, Randomized, Placebo-Controlled Crossover Trial of an Alpha-Lipoic Acid - Pregabalin Combination for the Treatment of Fibromyalgia Pain
}

Ian Gilron ${ }^{1}, \mathrm{MSc}, \mathrm{MD}$; Dongsheng $\mathrm{Tu}^{2}$, $\mathrm{PhD}$; Ronald Holden ${ }^{2}, \mathrm{PhD}$; Tanveer Towheed ${ }^{2}$, MD; Elizabeth Vandenkerkhof ${ }^{2}$, DrPH; Roumen $\mathrm{Milev}^{2}, \mathrm{MD}, \mathrm{PhD}$

${ }^{1}$ Queen's University, Department of Anesthesiology and Perioperative Medicine, Queen's University, Kingston, ON, Canada

${ }^{2}$ Queen's University, Kingston, ON, Canada

Corresponding Author:

Ian Gilron, MSc, MD

Queen's University

Department of Anesthesiology and Perioperative Medicine

Queen's University

Kingston General Hospital

76 Stuart Street, Victory 2 Pavillion

Kingston, ON,

Canada

Phone: 16135496666 ext 3963

Fax: 16135481375

Email: gilroni@queensu.ca

\begin{abstract}
Background: Fibromyalgia is a clinical disorder commonly presenting with chronic widespread pain as well as sleep disturbance, fatigue, depression, and cognitive dysfunction. There is an urgent need for treatment strategies that provide better pain relief and fewer adverse effects (AEs). Efforts to develop rational combinations of specific fibromyalgia treatments have demonstrated potential for measurable improvements in pain relief, quality of life, and health care utilization. More than half of fibromyalgia patients receive 2 or more analgesics but current combination use is based on limited evidence. As an early proof-of-concept project from the Canadian Institutes of Health Research-Strategy on Patient-Oriented Research Chronic Pain Network, this trial protocol is expected to advance the field by rigorously evaluating a new treatment combination for fibromyalgia.

Objective: We will test the hypothesis that analgesic combinations containing at least one nonsedating agent would be as safe but more effective than either monotherapy because of additive pain relief without increasing overall AEs. Pregabalin (PGB), a sedating anticonvulsant, is proven effective for fibromyalgia, and the antioxidant, alpha-lipoic acid (ALA), one of the only nonsedating systemic agents proven effective for neuropathic pain, is currently being evaluated in fibromyalgia. Thus, we will conduct a clinical trial to compare a PGB+ALA combination to each monotherapy for fibromyalgia.

Methods: Using a double-blind, double-dummy, crossover design, 54 adults with fibromyalgia will be randomly allocated to 1 of 6 sequences of treatment with PGB, ALA, and PGB+ALA combination. During each of 3 different treatment periods, participants will take 2 sets of capsules containing (1) ALA (or placebo) and (2) PGB (or placebo) for 31 days, followed by an 11-day taper/washout period. The primary outcome will be mean daily pain intensity ( 0 to 10 scale) at maximal tolerated doses (MTDs) during each period. Secondary outcomes, assessed at MTD, will include global improvement, adverse events, mood, and quality of life.
\end{abstract}

Results: This trial attained ethics approval March 6, 2017 (Queen's University Health Sciences and Affiliated Teaching Hospitals Research Ethics Board protocol number ANAE-313-17), and recruitment is set to start in August 2017.

Conclusions: This trial will provide rigorous evidence comparing the efficacy of a PGB-ALA combination to PGB alone and ALA alone in the treatment of fibromyalgia. 
Trial Registration: International Standard Randomized Controlled Trial Number ISRCTN14939460; https://www.isrctn.com/ ISRCTN1493946 (Archived by WebCite at http://www.webcitation.org/6sFqAjxkt)

(JMIR Res Protoc 2017;6(8):e154) doi: 10.2196/resprot.8001

\section{KEYWORDS}

fibromyalgia; alpha-lipoic acid; antioxidant; pregabalin; anticonvulsant

\section{Introduction}

Chronic pain affects $20 \%$ to $25 \%$ of the population [1] and is one of the most common reasons to see a health care provider and to miss work [2]. In North America alone, chronic pain costs over $\$ 650$ billion in health care and lost productivity [3]. Fibromyalgia is a complex clinical disorder characterized by chronic widespread pain that is also associated with sleep disturbance, fatigue, irritable bowel syndrome, depressed mood, and, possibly cognitive dysfunction [4,5]. Patients suffering with fibromyalgia very frequently report functional disability and impaired quality of life [6]; furthermore, fibromyalgia is a common disorder estimated to affect $1.6 \%$ of men and $4.9 \%$ of women [7].

Hundreds of randomized controlled trials (RCTs) have evaluated various drug (eg, nonsteroidal anti-inflammatory drugs, antidepressants, opioids, and anticonvulsants) and nondrug (eg, exercise, acupuncture, cognitive behavioral therapy) therapies for fibromyalgia [6]. In addition to exercise and cognitive behavioral therapy, pharmacotherapy remains an important treatment for fibromyalgia. Evidence-based treatment recommendations from various groups including the European League Against Rheumatism and Canadian Pain Society have included amitriptyline, cyclobenzaprine, tramadol, gabapentin/pregabalin, fluoxetine, and duloxetine [8-10].

Available drugs used for fibromyalgia reduce pain on average by only $25 \%$ to $40 \%$, and meaningful relief occurs in only $40 \%$ to $60 \%$ of patients, in part due to incomplete efficacy as well as commonly encountered dose-limiting adverse effects (AEs) (eg, sedation, cognitive dysfunction, and dizziness). Combining two drugs with different pharmacological mechanisms has the potential to provide superior relief over monotherapy without increasing side effects [11]. A recent trial has demonstrated greater analgesic efficacy with a pregabalin-duloxetine combination versus either monotherapy without an increase in side effect profile [12]. Although this was a positive finding, the additive benefit was submaximal because these two agents cause some similar AEs, and doses must be reduced during combination therapy to maintain safety and tolerability.

Thus, we hypothesize that analgesic combinations containing at least one nonsedating agent would provide even greater additive benefits because of additive pain relief but nonadditive AEs. Both pregabalin (PGB) and alpha-lipoic acid (ALA) are approved by Health Canada and proven for the treatment of neuropathic pain $[13,14]$. Based on a rationale for the use of
ALA to treat fibromyalgia pain [15], a placebo-controlled RCT of ALA monotherapy is currently under way in this population [ISRCTN58259979].

An important pharmacological mechanism of PGB is the blockade of $\alpha-2-\delta$ subunits of $N$-type voltage gated calcium channels, resulting in decreased calcium influx and neurotransmitter release [16,17]. ALA has been studied in both preclinical and clinical neuropathic pain conditions. In a rat model of streptozocin-induced diabetes, ALA delayed the onset of polyneuropathy [18]. Mechanistic studies suggest decreased nociceptive sensitivity by inhibition of T-type calcium (Cav3.2) channels [19], distinct from that of PGB which inhibits N-type calcium channels [17], suggesting potential for synergy at these different sites of action.

At least 16 trials of over 1320 patients have reported reductions in pain and other symptoms $[13,20]$ and a recent meta-analysis reported a number needed to treat of 6.3 [13]. Also, 1 trial reported improvement in neuropathic pain symptoms after 4 years of treatment [21]. AEs of nausea, vomiting, headache, and vertigo have been reported in studies involving >1200 mg per day of ALA. There have also been rare reports of hypoglycemia (low blood sugar) in diabetic patients taking ALA and reporting symptoms of sweating, paleness, chills, headache, dizziness, and/or confusion. We identified only 1 study of a combination similar to ALA+PGB-ALA plus gabapentin (related to PGB) in the treatment of burning mouth syndrome [22]. Despite the study having major methodological flaws, greater benefit with this combination was suggested versus monotherapy, and AEs were reported overall as very mild [22].

Thus, our goal is to conduct a novel double-blind RCT to compare the combination of the anticonvulsant PGB with the nonsedating antioxidant ALA to each monotherapy for the treatment of pain in fibromyalgia.

\section{Methods}

\section{Ethics}

This study underwent ethics review and received a compliance notice by the Queen's University Health Sciences and Affiliated Teaching Hospitals Research Ethics Board on March 6, 2017. This trial will be conducted at one site, Providence Care Hospital, Kingston, Ontario, Canada. This trial is registered with the International Standard Randomized Controlled Trial Number Registry [ISRCTN14939460]. 
Figure 1. Trial design.

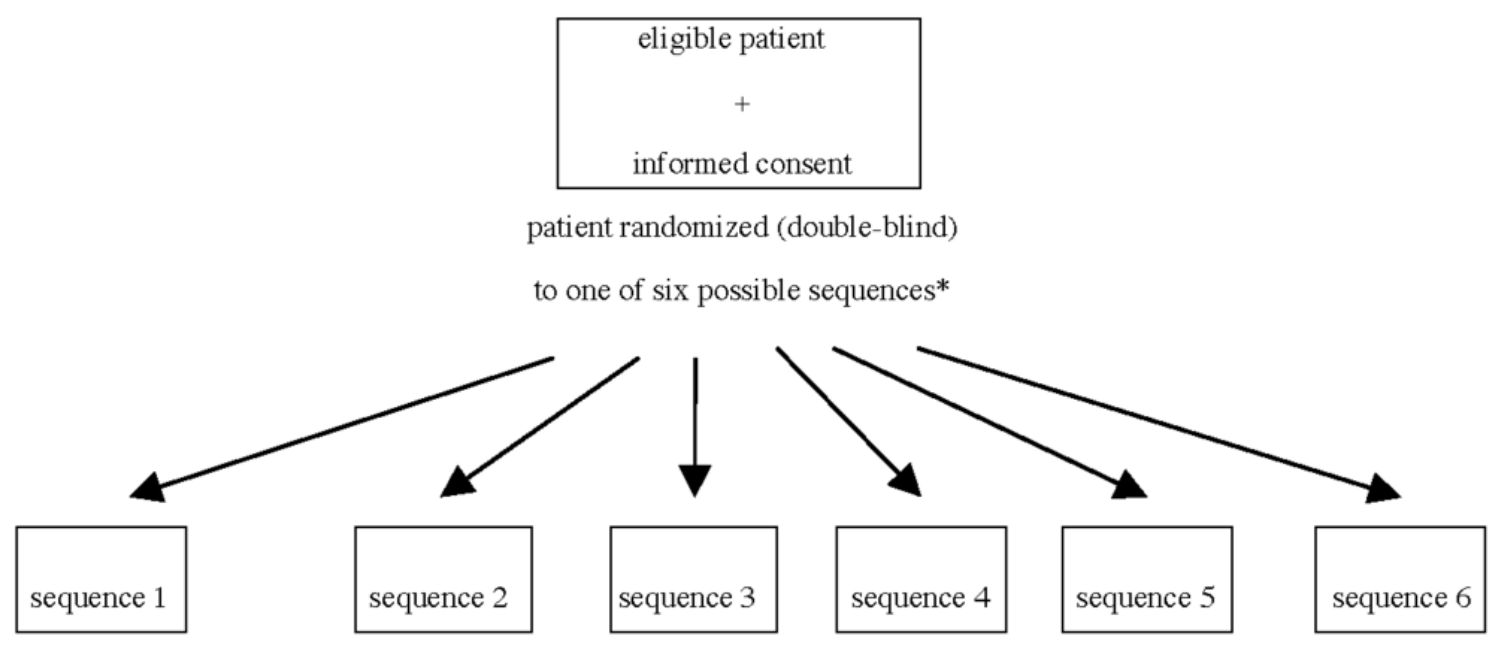

This will each be a 3-period, active treatment-controlled randomized double-blind trial, using a double-dummy, balanced Latin Square crossover design in which patients will be allocated to one of 6 treatment sequences of the three treatments: ALA, PGB and ALA-PGB combination

*see below for specific treatment sequences

\section{TREATMENT SEOUENCES}

(each patient is randomized to ONE of these sequences)

[All patients complete all three treatment periods (i.e. A, B and C) as per the treatment sequence they were randomized to (i.e. $1,2,3,4,5$ or 6 )]

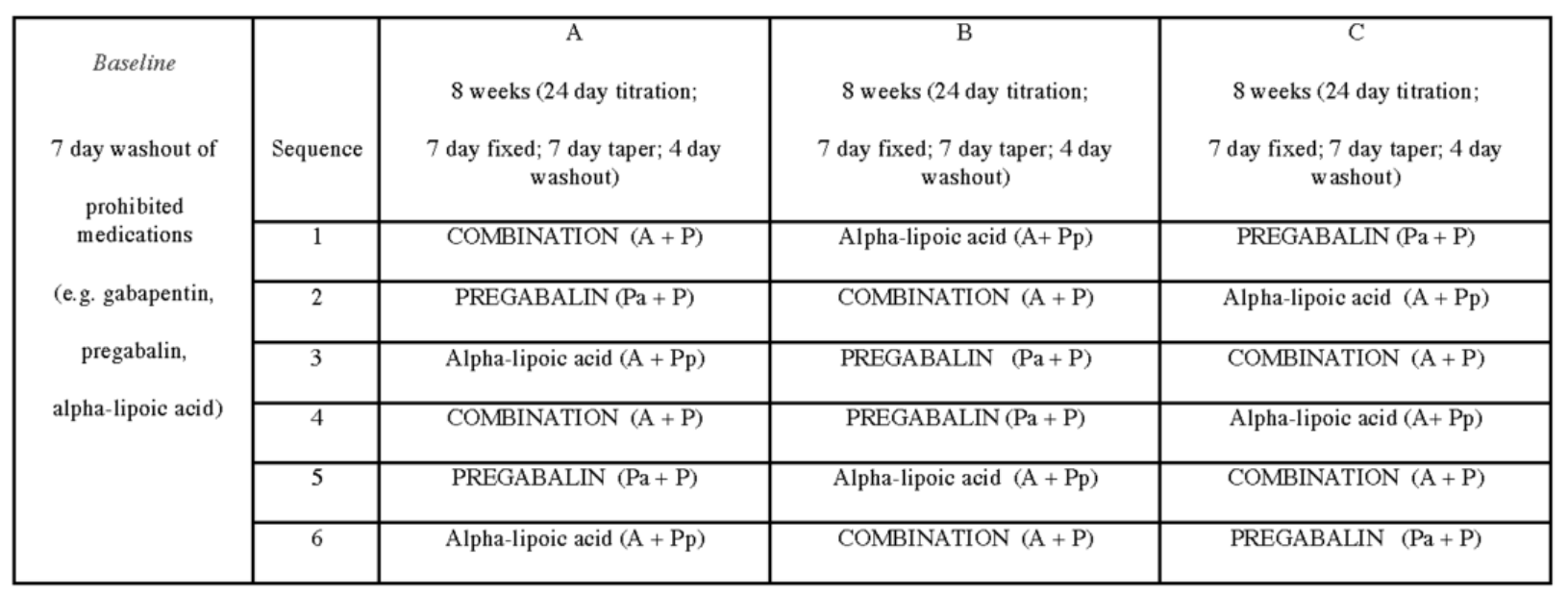

Legend: Symbols in parentheses indicate the content of the corresponding blinded study drug capsules as per the doubledummy design. $\mathrm{A}=$ =alpha-lipoic acid; $\mathrm{Pa}=$ "alpha-lipoic acid" placebo; $\mathrm{P}=$ pregabalin; $\mathrm{Pp}=$ "pregabalin" placebo

\section{Aims and Hypothesis}

The objective of this trial is to compare the safety and efficacy of a PGB+ALA combination to each monotherapy in treating participants with fibromyalgia. Our primary hypothesis is that PGB+ALA has greater analgesic efficacy versus either monotherapy.

\section{Trial Design}

We have designed a single-center, double-blind, double-dummy, randomized, controlled 3-period crossover trial comparing a PGB+ALA combination to monotherapy in treating fibromyalgia (Figures 1 and 2). This trial is compliant with Health Canada/International Conference on Harmonization guidelines and incorporates outcome measures recommended by the 
Initiative on Methods, Measurement, and Pain Assessment in Clinical Trials. Using a flexible dose titration, Latin Square crossover design, treatments will be titrated during each of 3 treatment periods to maximal tolerated dose (MTD) with primary and secondary trial analyses comparing the 3 treatments using end-of-period outcomes. Internal validity of our crossover design is supported by stability of fibromyalgia over time [23-26] and the risk of carryover from 1 period to the next is very low because each period is followed by an 11-day dose taper and drug washout, and the final MTD week for each period (from which the primary outcome is obtained) is separated from the next period's final week by 7 weeks (ie, $\geq 20$ half-lives of the drugs studied). Nevertheless, exploratory analyses will be conducted to identify if any low-order carryover effect does exist.

Figure 2. Trial design, continued.

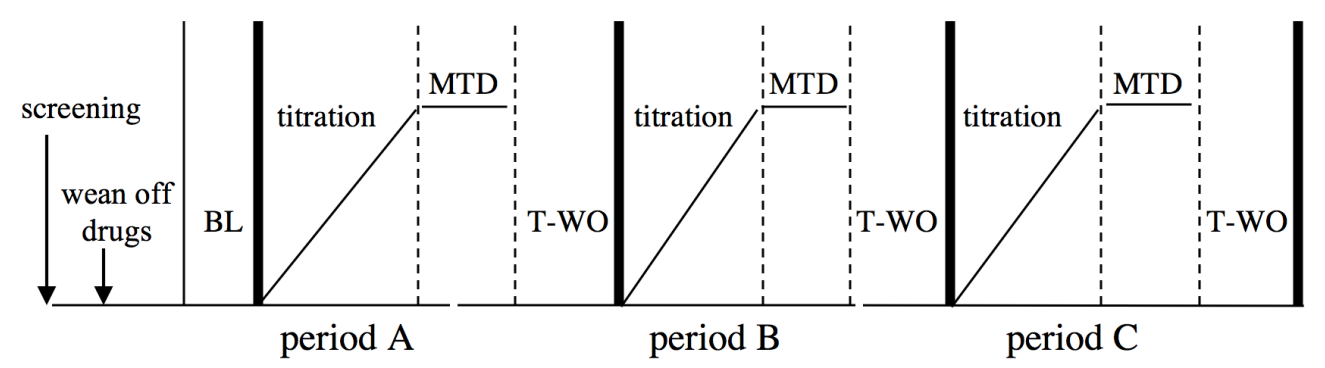

\begin{tabular}{|l|l|l|l|l|}
\hline 1 & 2 & 3 & 4 & 5 \\
\hline
\end{tabular}

\section{Screening}

2. Wean off current alpha-lipoic acid, gabapentin and pregabalin for $\geq 1$ week prior to commencing

- patients taking, and perceiving benefit from opioids ( $<90 /$ day morphine equivalents), antidepressants, nonsteroidal anti-inflammatory agents or acetaminophen may continue these at a steady dose during the study.

- patients required to avoid any procedural pain therapies (e.g. neurosurgical interventions, nerve blocks or acupuncture) during the entire study as these treatments may be unevenly distributed across treatment periods and could skew the study's results.

3. $\mathrm{BL}-7$ day baseline period

4. Double-blind randomization to one of six possible treatment sequences (e.g. sequence 1: combination>alpha-lipoic acid $>$ pregabalin) such that each patient progresses through each of three 6 week treatment periods (i.e. periods "A", "B" and "C").

5. Each treatment period starts with a 24 day study drug dose titration towards maximal tolerated dose (MTD). If MTD is reached before the end of this 24 day period, that dose will be continued up to and including days 25-31 of the treatment period and then concluded with a 7 day taper/ 4 day washout (T-WO).

A study nurse will contact patients by telephone at least twice a week to evaluate adverse effects, guide study drug titration and encourage compliance. Study patients will be encouraged to contact a study physician, as needed, who will be available 24 hours a day by pager in order to deal with study-related problems that may occur between scheduled study nurse telephone calls.

With each dosage increase of study medication in the titration schedule, if mild to moderate treatment-emergent adverse effects are encountered, patients will be asked if they can tolerate continuing at that dose for another 2-3 days. If so, this dosage will be continued with the expectation that tolerance to side effects will occur. If side effects are intolerable or do not improve, both study medications will be decreased to the next lowest possible dose and an increase will be attempted one more time at the next scheduled dose increase. If this again results in intolerable side effects, both study drugs will be decrease back to the previous dose, which will be defined as the maximally tolerated dose (MTD) for that individual.

\section{Participant Allocation}

As per the 3-period Latin Square crossover, patients are randomly allocated to 1 of 6 sequences of ALA, PGB, and combination (Figures 1 and 2). Before the trial, an independent pharmacist and biostatistician will prepare a concealed allocation schedule using a computer-generated block randomization process to randomly assign treatment sequences to a consecutive 
series of numbers within a block. Each patient will be assigned to the next consecutive number, and the corresponding sequence of medications will be dispensed. All study personnel will be blinded to the block sizes to preserve allocation concealment.

\section{Protecting Against Bias}

Medications will be encapsulated (ALA: blue, PGB: gray) in an identical fashion across all periods. As per a double-blind, double-dummy design, patients will take both sets of medications so treatment conditions will be identical across all 3 treatment periods. Treatment codes will be generated by the investigational pharmacist and concealed until trial completion.
In case of emergency, individual codes will be disclosed by an investigational pharmacist to a nonstudy clinician. A questionnaire completed by every participant at the end of each period will ask patients to guess the treatment they received to assess blinding.

\section{Participants}

Men and women aged 18 years and older meeting the 2016 updated American College of Rheumatology diagnostic criteria for fibromyalgia [27] will be considered for recruitment following informed consent. See Textbox 1 for selection criteria.

Textbox 1. Selection criteria.

Inclusion criteria:

- $\quad$ Adults aged 18 years and older

- Diagnosed with fibromyalgia using the 2016 updated American College of Rheumatology diagnostic criteria [27]

- $\quad$ Experience daily moderate pain $(\geq 4 / 10$ on a numerical rating scale) for at least 3 months

- Women of childbearing potential must have a negative serum beta-human chorionic gonadotropin test and are required to use a highly effective form of contraception while on trial

- Have the necessary abilities, visual acuity, and English language skills to complete questionnaires and pain diaries and to participate in telephone communication with study nurses to permit titration of the study drugs

Exclusion criteria:

- Presence of a painful condition, including inflammatory rheumatic disease, more than $50 \%$ as severe as but distinct from fibromyalgia

- Women who are pregnant or lactating

- Women of childbearing potential not using adequate contraceptives

- $\quad$ End-stage kidney or liver disease

- Unstable cardiovascular disease (myocardial infarction within the preceding year, unstable angina, or congestive heart failure) or clinically relevant abnormal 12-lead electrocardiogram

- Any poorly controlled medical condition that, in the opinion of the investigator, would interfere with proper conduct of the trial

- Severe depression, as determined by a Beck Depression Inventory-II score of 29 or more; suicidal ideation, as determined by a Beck Depression Inventory-II item 9 score of 2 or more; or any current major psychiatric disorder (eg, schizophrenia, bipolar disorder) that is not well controlled

- Hypersensitivity to any of the study medications

- Any current alcohol or drug abuse or dependence (except nicotine and caffeine). Participants with a history of abuse or dependence with more than 1 year of abstinence may be considered for inclusion

- $\quad$ Those taking more than $90 \mathrm{mg}$ morphine equivalents per day

\section{Study Interventions}

During each of 3 trial periods, using a double-blind randomized crossover design, patients will receive 2 sets of capsules (Figure 3): (1) blue capsules (ALA $300 \mathrm{mg}$ or placebo) and (2) gray capsules (PGB $75 \mathrm{mg}$ or placebo). During the combination period, blue will contain ALA and gray will contain PGB. During the ALA alone period, blue will contain ALA and gray will contain placebo. During the PGB alone period, blue will contain placebo and gray will contain PGB.

Consenting patients on ALA or PGB (or gabapentin) pretrial will agree to be weaned gradually for a washout of at least 7 days. Co-interventions: patients taking and perceiving benefit from opioids (<90 mg morphine equivalents), antidepressants (tricyclic, selective serotonin reuptake inhibitors or serotonin-norepinephrine reuptake inhibitors), nonsteroidal anti-inflammatory drugs, or acetaminophen may continue these at a steady dose for the entire study. Any cognitive behavioral therapy or exercise programs may continue only if they can be scheduled evenly across all treatment periods. Research staff will monitor and advise patients weekly about prohibited co-interventions throughout the study. A thorough understanding of the threats to validity of using forbidden co-interventions (gabapentin, PGB, ALA, and any other newly initiated analgesic intervention) is heavily emphasized to participants. Patients will not be allowed to start new cognitive behavioral therapy or exercise programs after study initiation and must avoid any procedural therapies (eg, nerve blocks or acupuncture) during the entire study. Any pain exacerbations that in the opinion of the patient warrant initiation of a new therapy would necessitate trial discontinuation and immediate weaning from study 
medications, but these patients would still be included in the trial analyses.

\section{Dose Titration}

Study medication will follow a flexible dose titration to MTD to balance tolerability and relief, with regularly weekly calls by research personnel. This means that doses of study medication will not be further increased if intolerable AEs are encountered at higher doses or if "a lot" or "complete" pain relief is achieved. The MTD fixed dose week will be from days 25 to 31 . However, if MTD is reached before day 25, that MTD dose will continue up to and including the day 25 to 31 period. The MTD fixed dose week will be followed by a 7-day dose taper and 4-day complete washout. Daily pain ratings will be completed throughout the trial. During dose taper and washout periods only, patients may take acetaminophen $\leq 8$ tablets per day ( 325 mg per tablet) as needed. This rescue medication is very unlikely to affect the primary outcome measure of pain intensity during the MTD phase of each treatment period.

\section{Trial Duration and Follow-Up Frequency}

Each of the 3 treatment periods will be 6 weeks, for a total trial duration of 18 weeks. The nurse will phone patients weekly to evaluate AEs, guide drug titration, and encourage compliance. Patients will be seen in clinic at the end of each treatment period for assessment of vital signs and measurement of secondary outcomes (Figure 4). Patients will be followed up by phone 2 weeks and 3 months after trial completion (including patients who were withdrawn from the trial prematurely) to document any subsequent AEs.

Figure 3. Study drug schedules.

$$
\text { A= "alpha-lipoic acid" (ALA or placebo); P= "pregabalin" (PGB or placebo) }
$$

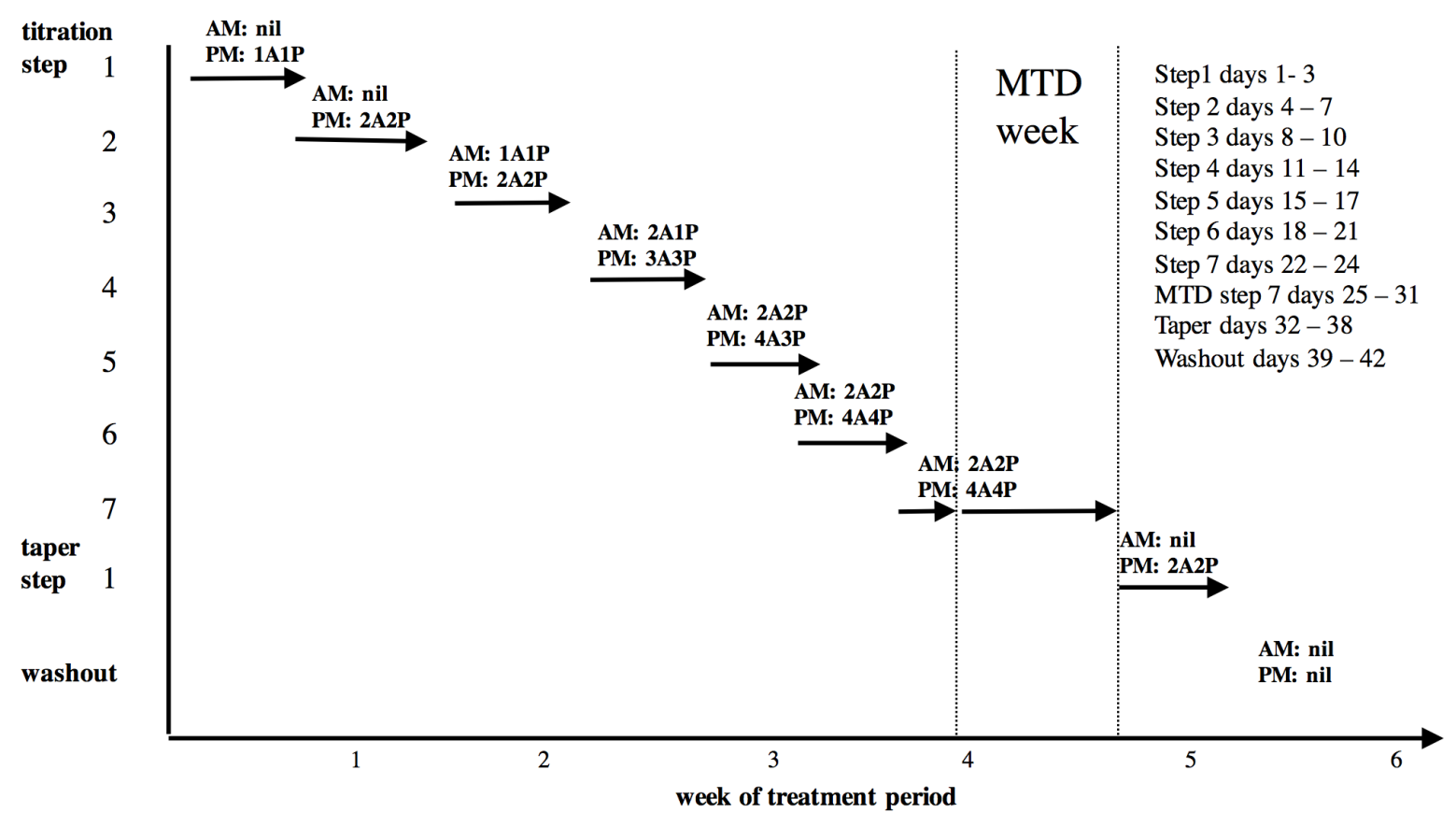

For each trial period, patients receive blue "A" (ALA 300 or placebo), and grey "P" (PGB 75mg or placebo), capsules. Each step above indicates the number of each capsules taken before breakfast $\&$ in the evening. The schedule above indicates uppermost dose ceilings at each timepoint. Titration towards individual maximally tolerated dose (MTD) will be guided by safety determined by weekly AE monitoring. Thus, titration may be slower and MTD may be lower than shown above.

\section{Outcome Measures and Safety Assessment}

The primary outcome is mean daily pain (0 to 10 numerical rating scale with $0=$ no pain and $10=$ worst pain imaginable), rated 3 times daily ( $8 \mathrm{AM}, 4 \mathrm{PM}$, and $8 \mathrm{PM}$ ) and averaged over the MTD fixed dose week (days 39 to 45 ) of each period.
Secondary outcomes include daily pain at other timepoints, the MTDs of PGB and ALA, frequency and severity of AEs and patient global impression of change [28], short form McGill Pain Questionnaire [29], Fibromyalgia Impact Questionnaire [30], Brief Pain Inventory [31], Beck Depression Inventory-II [32], Beck Anxiety Inventory [33], Short Form-36 health survey 
[34], blinding questionnaires, and acetaminophen consumption. Timing of outcome assessments is described in Figure 4. Patient safety will be ensured by vigilant $\mathrm{AE}$ assessment and judicious drug titration. Any occurrences of major AEs will be tracked as secondary outcomes and also reported to the Queen's Ethics Board, Health Canada. Assessment and reporting of AEs will adhere to Consolidated Standards for Reporting Trials recommendations [35].

Figure 4. Schedule of assessments.

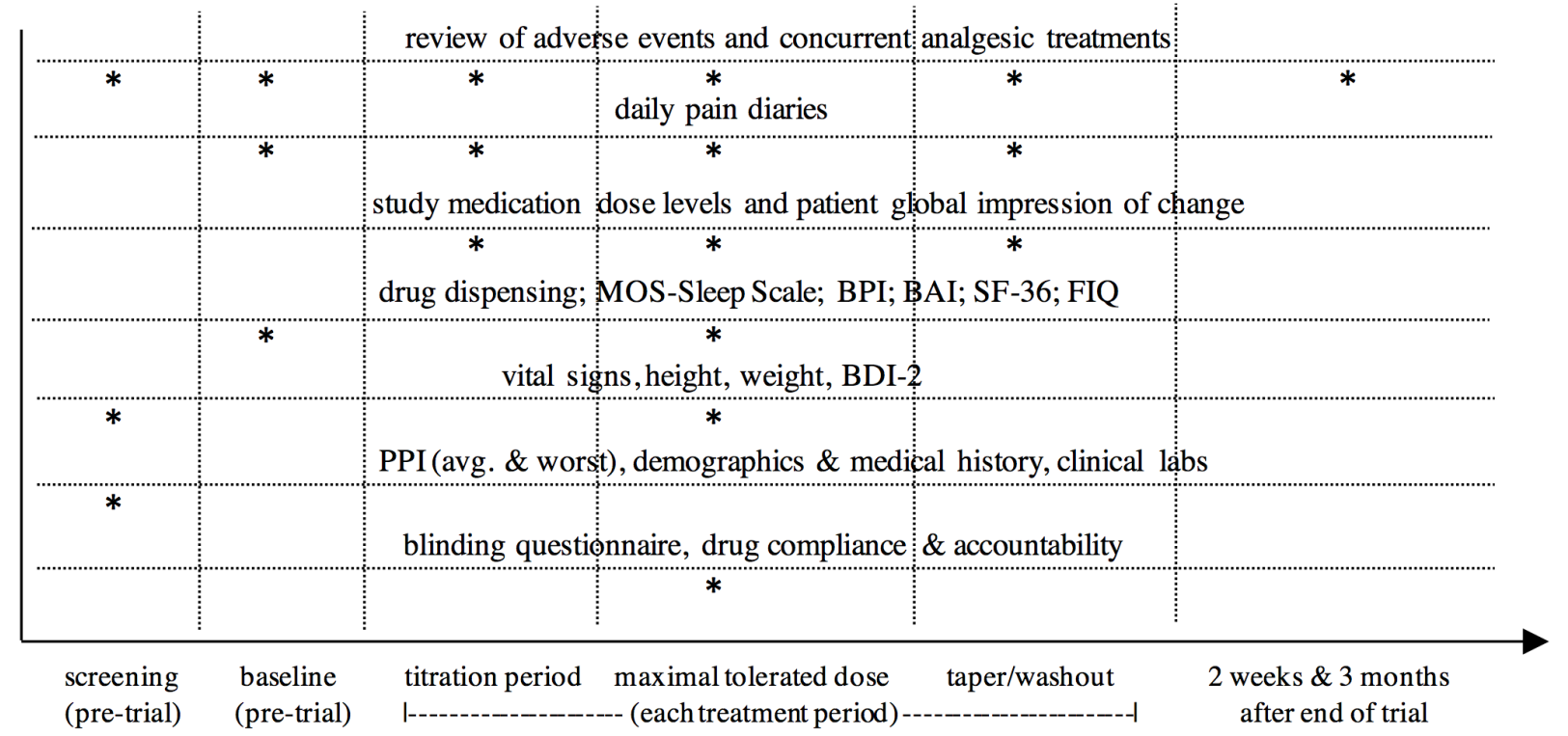

PPI - present pain intensity (0-10 numerical scale) MOS - Medical Outcomes Study

SF-MPQ - short form McGill Pain Questionnaire; BPI - Brief Pain Inventory;

\section{Sample Size}

Based on previous estimates of within-patient variation, $s=2.45$, from a previous study in fibromyalgia [36], we calculate that a sample of 55 trial completers would provide an $80 \%$ chance of detecting (alpha of .05) a mean treatment difference of 1 point ( 0 to 10 scale). For a sample size divisible by 6 , the number of treatment sequences, we adjusted the sample size to 54 patients.

\section{Statistical Analyses}

Analyses for this trial are based on the null hypothesis of no difference between PGB, ALA, and PGB+ALA, and the alternative hypothesis that at least 2 treatments are different. When a patient contributes data from only 1 period, sensitivity analyses including all patients will also be performed by assuming some reasonable but extreme values for the remaining periods. All patients receiving at least 1 dose of a drug will be included in the safety analyses.

\section{Primary Outcome Analysis}

The primary outcome-mean daily pain from the last 7 days (at MTD) of each treatment period-will be calculated as an average of pain scores as recorded in the pain diary if more than $50 \%$ of the information (ie, at least 4 days) is not missing. Otherwise, mean daily pain will be treated as missing. This is based on the half rule often used to summarize repeated
BDI-2 - Beck Depression Inventory - 2;

BAI - Beck Anxiety Inventory;

SF-36 - the MOS 36-item short-form health survey

FIQ - Fibromyalgia Impact Questionnaire

responses, which has proven unlikely to introduce bias to trial results [37]. Sensitivity analyses based on the average of all available pain scores will also be performed to confirm the results of the primary analysis. Although carryover effects are unlikely, we recognize this possibility. Therefore, a linear mixed model with sequence, period, treatment, and the first order carryover term as fixed effects and patient as a random effect [38] will be used to test for differences among the 3 treatments and to estimate the least square mean of the mean daily pain intensity for each treatment, adjusting for carryover as well as period effects (ie, stability of pain levels). The following 3 pair-wise comparisons will be performed based on the least square means and standard deviations from the linear mixed model: combination versus ALA alone, combination versus PGB alone, and ALA alone versus PGB alone. Sensitivity analyses will be performed using a pattern-mixture model [39] based on patterns of missing data so as to check the robustness of results in the case that data may not be missing at random. A Fisher's least significant difference [40] procedure will be used to adjust the $P$ values for these 3 comparisons.

\section{Secondary Analyses}

Secondary outcomes will be analyzed similarly except that only one measurement is analyzed in the last week for the singular measures (ie, final week questionnaires) and the scoring 
algorithms developed for the Brief Pain Inventory, Beck Depression Inventory-II, and Short Form-36 will be first used to derive the subscales or domains within these instruments, and the scores on these subscales or domains will be used as response variables in the linear mixed model analysis.

As with many other analgesic trials that allow concomitant medications, treatment group comparisons are made with the assumption in the setting of randomization of an equal distribution of concomitant medications across participants. Nevertheless, our trial analysis will further conduct exploratory analyses, as we have done on our previous trials, to investigate the possibility that concomitant analgesic medications have an important effect on the trial results.

\section{Results}

Participant recruitment is expected to begin in August 2017. This trial was awarded external peer-reviewed funding by the Canadian Institutes of Health Research-Strategy on Patient-Oriented Research-Canadian Pain Network in August 2016.

\section{Discussion}

Fibromyalgia continues to be difficult to manage, and current treatments provide only partial relief often at the risk of disabling AEs. To the best of our knowledge, this proposed trial is the first to compare the combination of an anticonvulsant with an antioxidant to treat fibromyalgia. Because ALA and PGB have different AE profiles, we expect their combination to provide superior analgesic efficacy in fibromyalgia without increasing AEs.

Possible threats to trial completion include challenges with participant recruitment, noncompliance, protocol violations, and early dropouts. However, we are confident that the proposed trial design and our experience with recent and previous RCTs will minimize these concerns. Noncompliance, protocol violations, and early dropouts will be minimized by the crossover design as well as thorough patient teaching and careful follow-up of trial participants.

Given the urgent need for improved fibromyalgia treatments that provide better pain relief with better safety and tolerability, this trial will provide rigorous evidence for a potentially improved treatment strategy for fibromyalgia.

\section{Acknowledgments}

The authors thank the Canadian Institutes of Health Research and the Queen's University Department of Anesthesiology and Perioperative Medicine.

\section{Authors' Contributions}

All authors read and approved the manuscript. IG led the writing of this manuscript and the development of this protocol. DT led the development of the statistical analysis plan and contributed to writing of this manuscript and development of the protocol. RRH participated in the writing of this manuscript and initial protocol development, including selection of mood and quality of life measures. TT participated in the initial protocol development. EV participated in the writing of this manuscript and initial protocol development. RM participated in the writing of this manuscript and initial protocol development. All authors will be involved in data analysis and interpretation and manuscript preparation.

\section{Conflicts of Interest}

IG has received support from Biogen, Adynxx, TARIS Biomedical, AstraZeneca, Pfizer, and Johnson and Johnson and has received grants from the Canadian Institutes of Health Research, Physicians' Services Incorporated Foundation, and Queen's University. RRH has received research funding from the Canadian Institutes of Health Research, the Social Sciences and Humanities Research Council of Canada, the American Foundation for Suicide Prevention, and Queen's University. The remaining authors have no conflicts of interest to declare.

\section{Multimedia Appendix 1}

Peer reviewer comments (CIHR-reviews-CADENCE-funded).

[PDF File (Adobe PDF File), 133KB-Multimedia Appendix 1]

\section{References}

1. Reitsma ML, Tranmer JE, Buchanan DM, Vandenkerkhof EG. The prevalence of chronic pain and pain-related interference in the Canadian population from 1994 to 2008. Chronic Dis Inj Can 2011 Sep;31(4):157-164 [FREE Full text] [Medline: 21978639]

2. St Sauver JL, Warner DO, Yawn BP, Jacobson DJ, McGree ME, Pankratz JJ, et al. Why patients visit their doctors: assessing the most prevalent conditions in a defined American population. Mayo Clin Proc 2013 Jan;88(1):56-67 [FREE Full text] [doi: 10.1016/j.mayocp.2012.08.020] [Medline: 23274019]

3. Institute of Medicine. Relieving Pain in America: A Blueprint for Transforming Prevention, Care, Education, and Research. Washington: National Academies Press; 2011. 
4. Wolfe F. Fibromyalgia wars. J Rheumatol 2009 Apr;36(4):671-678. [doi: 10.3899/jrheum.081180] [Medline: 19342721]

5. Gelonch O, Garolera M, Valls J, Rosselló L, Pifarré J. Cognitive complaints in women with fibromyalgia: are they due to depression or to objective cognitive dysfunction? J Clin Exp Neuropsychol 2017 Mar 17:1-13. [doi: 10.1080/13803395.2017.1301391] [Medline: 28301977]

6. Clauw DJ. Fibromyalgia: a clinical review. JAMA 2014 Apr 16;311(15):1547-1555. [doi: 10.1001/jama.2014.3266] [Medline: 24737367]

7. Gran JT. The epidemiology of chronic generalized musculoskeletal pain. Best Pract Res Clin Rheumatol 2003 Aug;17(4):547-561. [Medline: 12849711]

8. Fitzcharles M, Ste-Marie PA, Goldenberg DL, Pereira JX, Abbey S, Choinière M, National Fibromyalgia Guideline Advisory Panel. 2012 Canadian Guidelines for the diagnosis and management of fibromyalgia syndrome: executive summary. Pain Res Manag 2013;18(3):119-126 [FREE Full text] [Medline: 23748251]

9. Thieme K, Mathys M, Turk DC. Evidenced-based guidelines on the treatment of fibromyalgia patients: are they consistent and if not, why Not? Have Effective Psychological Treatments Been Overlooked? J Pain 2016 Dec 27. [doi: 10.1016/j.jpain.2016.12.006] [Medline: 28034828]

10. Macfarlane GJ, Kronisch C, Dean LE, Atzeni F, Häuser W, Fluß E, et al. EULAR revised recommendations for the management of fibromyalgia. Ann Rheum Dis 2017 Feb;76(2):318-328. [doi: 10.1136/annrheumdis-2016-209724] [Medline: $\underline{27377815}$

11. Mease PJ, Seymour K. Fibromyalgia: should the treatment paradigm be monotherapy or combination pharmacotherapy? Curr Pain Headache Rep 2008 Dec;12(6):399-405. [Medline: 18973731]

12. Gilron I, Chaparro LE, Tu D, Holden RR, Milev R, Towheed T, et al. Combination of pregabalin with duloxetine for fibromyalgia: a randomized controlled trial. Pain 2016 Dec;157(7):1532-1540. [doi: 10.1097/j.pain.0000000000000558] [Medline: 26982602]

13. Ziegler D, Nowak H, Kempler P, Vargha P, Low PA. Treatment of symptomatic diabetic polyneuropathy with the antioxidant alpha-lipoic acid: a meta-analysis. Diabet Med 2004 Feb;21(2):114-121. [Medline: 14984445]

14. Finnerup NB, Attal N, Haroutounian S, McNicol E, Baron R, Dworkin RH, et al. Pharmacotherapy for neuropathic pain in adults: a systematic review and meta-analysis. Lancet Neurol 2015 Feb;14(2):162-173 [FREE Full text] [doi: 10.1016/S1474-4422(14)70251-0] [Medline: 25575710]

15. Gilron I, Tu D, Holden R, Towheed T, Ziegler D, Wang L, et al. Innovations in the management of musculoskeletal pain with alpha-lipoic acid (IMPALA trial): study protocol for a double-blind, randomized, placebo-controlled crossover trial of alpha-lipoic acid for the treatment of fibromyalgia pain. JMIR Res Protoc 2017 Mar 28;6(3):e41 [FREE Full text] [doi: 10.2196/resprot.7198] [Medline: 28351829]

16. Luo ZD, Chaplan SR, Higuera ES, Sorkin LS, Stauderman KA, Williams ME, et al. Upregulation of dorsal root ganglion (alpha)2(delta) calcium channel subunit and its correlation with allodynia in spinal nerve-injured rats. J Neurosci 2001 Mar 15;21(6):1868-1875 [FREE Full text] [Medline: 11245671]

17. Taylor CP, Angelotti T, Fauman E. Pharmacology and mechanism of action of pregabalin: the calcium channel alpha2-delta (alpha2-delta) subunit as a target for antiepileptic drug discovery. Epilepsy Res 2007 Feb;73(2):137-150. [doi: 10.1016/j.eplepsyres.2006.09.008] [Medline: 17126531]

18. Nagamatsu M, Nickander KK, Schmelzer JD, Raya A, Wittrock DA, Tritschler H, et al. Lipoic acid improves nerve blood flow, reduces oxidative stress, and improves distal nerve conduction in experimental diabetic neuropathy. Diabetes Care 1995 Aug;18(8):1160-1167. [Medline: 7587852]

19. Lee WY, Orestes P, Latham J, Naik AK, Nelson MT, Vitko I, et al. Molecular mechanisms of lipoic acid modulation of T-type calcium channels in pain pathway. J Neurosci 2009 Jul 29;29(30):9500-9509 [FREE Full text] [doi: 10.1523/JNEUROSCI.5803-08.2009] [Medline: 19641113]

20. Han T, Bai J, Liu W, Hu Y. A systematic review and meta-analysis of alpha-lipoic acid in the treatment of diabetic peripheral neuropathy. Eur J Endocrinol 2012 Oct;167(4):465-471 [FREE Full text] [doi: 10.1530/EJE-12-0555] [Medline: 22837391]

21. Ziegler D, Low PA, Litchy WJ, Boulton AJM, Vinik AI, Freeman R, et al. Efficacy and safety of antioxidant treatment with alpha-lipoic acid over 4 years in diabetic polyneuropathy: the NATHAN 1 trial. Diabetes Care 2011 Sep;34(9):2054-2060 [FREE Full text] [doi: 10.2337/dc11-0503] [Medline: 21775755]

22. López-D'alessandro E, Escovich L. Combination of alpha lipoic acid and gabapentin, its efficacy in the treatment of Burning Mouth Syndrome: a randomized, double-blind, placebo controlled trial. Med Oral Patol Oral Cir Bucal 2011 Aug 01;16(5):e635-e640 [FREE Full text] [Medline: 20711135]

23. Crofford LJ, Mease PJ, Simpson SL, Young JP, Martin SA, Haig GM, et al. Fibromyalgia relapse evaluation and efficacy for durability of meaningful relief (FREEDOM): a 6-month, double-blind, placebo-controlled trial with pregabalin. Pain 2008 Jun;136(3):419-431. [doi: 10.1016/j.pain.2008.02.027] [Medline: 18400400]

24. Felson DT, Goldenberg DL. The natural history of fibromyalgia. Arthritis Rheum 1986 Dec;29(12):1522-1526. [Medline: 3801074]

25. Forseth KO, Førre O, Gran JT. A 5.5 year prospective study of self-reported musculoskeletal pain and of fibromyalgia in a female population: significance and natural history. Clin Rheumatol 1999;18(2):114-121. [Medline: 10357115] 
26. Kennedy M, Felson DT. A prospective long-term study of fibromyalgia syndrome. Arthritis Rheum 1996 Apr;39(4):682-685. [Medline: $\underline{8630121]}$

27. Wolfe F, Clauw DJ, Fitzcharles M, Goldenberg DL, Häuser W, Katz RL, et al. 2016 revisions to the 2010/2011 fibromyalgia diagnostic criteria. Semin Arthritis Rheum 2016 Dec;46(3):319-329. [doi: 10.1016/j.semarthrit.2016.08.012] [Medline: 27916278]

28. Farrar JT, Young JP, LaMoreaux L, Werth JL, Poole RM. Clinical importance of changes in chronic pain intensity measured on an 11-point numerical pain rating scale. Pain 2001 Nov;94(2):149-158. [Medline: 11690728]

29. Melzack R. The short-form McGill Pain Questionnaire. Pain 1987 Aug;30(2):191-197. [Medline: $\underline{3670870]}$

30. Burckhardt CS, Clark SR, Bennett RM. The fibromyalgia impact questionnaire: development and validation. J Rheumatol 1991 May;18(5):728-733. [Medline: 1865419]

31. Cleeland CS, Ryan KM. Pain assessment: global use of the Brief Pain Inventory. Ann Acad Med Singapore 1994 Mar;23(2):129-138. [Medline: $\underline{8080219}$ ]

32. Beck A, Steer R, Brown G. BDI-II: Beck Depression Inventory Manual, 2nd Edition. San Antonio: Harcourt Brace and Company; 1996.

33. Steer RA, Kumar G, Ranieri WF, Beck AT. Use of the Beck Anxiety Inventory with adolescent psychiatric outpatients. Psychol Rep 1995 Apr;76(2):459-465. [doi: 10.2466/pr0.1995.76.2.459] [Medline: 7667457]

34. Ware J, Snow K, Kosinski MB. SF-36 Health Survey Manual and Interpretation Guide. Boston: The Health Institute; 1993.

35. Ioannidis JPA, Evans SJW, Gøtzsche PC, O'Neill RT, Altman DG, Schulz K, CONSORT Group. Better reporting of harms in randomized trials: an extension of the CONSORT statement. Ann Intern Med 2004 Nov 16;141(10):781-788. [Medline: $\underline{15545678]}$

36. Russell IJ, Mease PJ, Smith TR, Kajdasz DK, Wohlreich MM, Detke MJ, et al. Efficacy and safety of duloxetine for treatment of fibromyalgia in patients with or without major depressive disorder: Results from a 6-month, randomized, double-blind, placebo-controlled, fixed-dose trial. Pain 2008 Jun;136(3):432-444. [doi: 10.1016/j.pain.2008.02.024] [Medline: 18395345]

37. Fairclough D. Analyzing studies with missing data. In: Fairclough D, Fayers PM, Hays RD, editors. Assessing Quality of Life in Clinical Trials, 2nd Edition. Oxford: Oxford University Press; 2005:182.

38. Jones B, Kenward MG. Design and Analysis of Cross-Over Trials, 3rd Edition. Boca Raton: CRC Press; 2015.

39. Molenberghs G, Kenward MG. Missing Data in Clinical Studies. Hoboken: Wiley; 2007.

40. Hochberg Y, Tamhane A. Multiple Comparison Procedures. New York: Wiley; 1987.

\section{Abbreviations}

AE: adverse effect

ALA: alpha-lipoic acid

MTD: maximal tolerated dose

PGB: pregabalin

RCT: randomized controlled trial

Edited by G Eysenbach; submitted 08.05.17; peer-reviewed by H Shanthana; comments to author 14.07.17; revised version received 19.07.17; accepted 21.07.17; published 04.08.17

\section{Please cite as:}

Gilron I, Tu D, Holden R, Towheed T, Vandenkerkhof E, Milev R

Combination Analgesic Development for Enhanced Clinical Efficacy (CADENCE Trial): Study Protocol for a Double-Blind, Randomized, Placebo-Controlled Crossover Trial of an Alpha-Lipoic Acid - Pregabalin Combination for the Treatment of Fibromyalgia Pain JMIR Res Protoc 2017;6(8):e154

URL: http://www.researchprotocols.org/2017/8/e154/

doi: 10.2196/resprot.8001

PMID: 28778847

CIan Gilron, Dongsheng Tu, Ronald Holden, Tanveer Towheed, Elizabeth Vandenkerkhof, Roumen Milev. Originally published in JMIR Research Protocols (http://www.researchprotocols.org), 04.08.2017. This is an open-access article distributed under the terms of the Creative Commons Attribution License (https://creativecommons.org/licenses/by/4.0/), which permits unrestricted use, distribution, and reproduction in any medium, provided the original work, first published in JMIR Research Protocols, is properly cited. The complete bibliographic information, a link to the original publication on http://www.researchprotocols.org, as well as this copyright and license information must be included. 